\title{
Infrared Action Spectroscopy of Low-Temperature Neutral Gas-Phase Molecules of Arbitrary Structure
}

\author{
Vasyl Yatsyna, ${ }^{1,2}$ Daniël J. Bakker, ${ }^{2}$ Peter Salén, ${ }^{3}$ Raimund Feifel, ${ }^{1}$ Anouk M. Rijs, ${ }^{2, *}$ and Vitali Zhaunerchyk ${ }^{1, \dagger}$ \\ ${ }^{1}$ Department of Physics, University of Gothenburg, 41296 Gothenburg, Sweden \\ ${ }^{2}$ Radboud University, Institute for Molecules and Materials, FELIX Laboratory, Toernoovield 7-c, 6525 ED Nijmegen, The Netherlands \\ ${ }^{3}$ Department of Physics, Stockholm University, 10691 Stockholm, Sweden
}

(Received 11 May 2016; published 9 September 2016)

\begin{abstract}
We demonstrate a technique for IR action spectroscopy that enables measuring IR spectra in a background-free fashion for low-temperature neutral gas-phase molecules of arbitrary structure. The method is exemplified experimentally for $N$-methylacetamide molecules in the mid-IR spectral range of $1000-1800 \mathrm{~cm}^{-1}$, utilizing the free electron laser FELIX. The technique involves the resonant absorption of multiple mid-IR photons, which induces molecular dissociation. The dissociation products are probed with $10.49 \mathrm{eV}$ vacuum ultraviolet photons and analyzed with a mass spectrometer. We also demonstrate the capability of this method to record, with unprecedented ease, mid-IR spectra for the molecular associates, such as clusters and oligomers, present in a molecular beam. In this way the mass-selected spectra of lowtemperature gas-phase dimers and trimers of $N$-methylacetamide are measured in the full amide I-III range.
\end{abstract}

DOI: $10.1103 /$ PhysRevLett.117.118101

The two central topics of modern biophysics are biomolecular structure elucidation and obtaining insight into processes relevant for biomolecular functioning. These two topics are closely related to each other as in many cases the structure of biomolecules determines their functioning. Examples of this relation include molecular recognition, the selective binding of ligands, the regulation of protein activity upon conformational changes, etc. On the contrary, the faulty structures of biomolecules hamper their proper functioning, which is the case for certain neurodegenerative diseases associated with the misfolding of proteins $[1,2]$. An overall insight into the structure and processes occurring in large biomolecules can often be obtained by separately investigating their building blocks or essential parts, such as individual amino acids, peptides, nuclear bases, etc. [3,4]. The advantages of studying such relatively simple biologically relevant molecular systems are that they can easily be handled in the gas phase, which enables studies of the intrinsic properties without the influence of neighboring molecules. Furthermore, they are amenable to sophisticated theoretical calculations.

Mid-IR and terahertz spectroscopy methods are highly suitable for (bio)molecular structure elucidation [4-8]. These techniques are in particular capable of unraveling even small changes in molecular structure $[6,9,10]$. They also enable the probing of the hydrogen bonds $[4,11,12]$ that play a significant role in, e.g., the folding of proteins into higher-order structures. Free electron lasers (FELs) are distinct among other existing mid-IR and terahertz sources due to their capability of generating highly intense wavelength-tunable monochromatic light. Such FELs typically generate light in the form of microsecond long macropulses, which in turn consist of subnanosecond long micropulses $[13,14]$. As the number of gas-phase molecules in the interaction region is significantly smaller than the number of photons in the FEL pulses, direct absorption measurements are very challenging and, instead, action spectroscopy techniques are implemented. IR action spectroscopy implies measuring signals associated with IR photon absorptions in the form of, for example, electron or ion signals, versus different IR wavelengths. Currently, there are two methods routinely employed in IR FELs to measure the spectra in this spectral region for charged and neutral molecular species. One is based on IR multiphoton dissociation (MPD) [15-17] and is widely employed for both cations and anions [18-22]. Upon MPD, the resonant absorption of successive photons is intermediated by intramolecular vibrational energy redistribution (IVR) [17]. The rate of IVR determines the time span needed for the molecule to reach a dissociative state. In this approach, mass selected charged molecules are stored in a trap, where they are irradiated with infrared light. To measure the spectrum for a neutral molecule the approach of IR-UV ion-dip spectroscopy [23] is typically used. The limitation of the latter is that it is only applicable for molecules possessing an UV chromophore (e.g., an aromatic ring) to absorb the UV light. Currently, there is no general method routinely implemented to study neutral molecules in the gas phase that do not possess an UV chromophore.

In this Letter, we demonstrate a method to measure IR spectra for molecules of arbitrary structure utilizing FEL radiation sources. This approach can be considered as a two-color pump-probe measurement, in which intense IR light is utilized to start the MPD reaction, i.e., to resonantly pump the molecule until it dissociates, while vacuum 


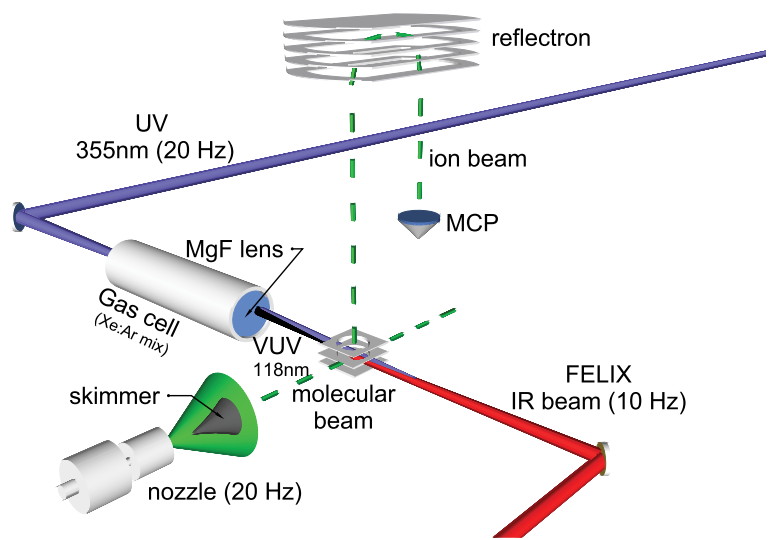

FIG. 1. Schematic representation of the experimental setup for the IR action spectroscopy of low-temperature neutral gas-phase molecules. The NMA sample was heated to $90{ }^{\circ} \mathrm{C}$, mixed with the carrier gas argon ( 3 bar) and expanded into vacuum to induce supersonic-jet cooling. The skimmed molecular beam was introduced into the interaction region where it overlapped with the FELIX IR pulses. The IR photons were resonantly absorbed leading to MPD. The MPD products were ionized with VUV $(10.49 \mathrm{eV})$ pulses and the ionized species were analyzed with a time-of-flight reflectron-type mass spectrometer.

ultraviolet (VUV) ionizing light probes the MPD products. The advantages of this new method are (1) it enables measuring IR spectra for molecules of arbitrary structure, (2) the obtained IR spectra are background-free, (3) the measurements are performed for molecules embedded into supersonic beams, which ensures the cooling of the internal degrees of freedom down to 10-20 K, and (4) the IR spectra of the molecular clusters and oligomers present in the molecular beam can be measured simultaneously with the spectra for the parent molecules. In contrast to IR-UV iondip spectroscopy, this method does not have conformer selectivity. Thus, the measured IR spectra contain contributions of all conformations present in the molecular beam, weighted by their corresponding populations.

The experimental studies were performed at the free electron laser FELIX laboratory located at Radboud University, The Netherlands. The experimental setup, described in detail in Ref. [23], is adapted as presented in Fig. 1. The investigated molecules were $\mathrm{N}$-methylacetamide (NMA, see Fig. 2) and its fully deuterated isotopologue (NMA- $d 7$ ), which were prepared commercially with a purity $>98 \%$. The molecules were cooled in a supersonic jet expansion, skimmed, and delivered into the interaction region in the form of a pulsed collimated molecular beam. The longest possible interaction time between the molecules and the IR light was determined by the FELIX macropulse duration, which is typically $4-5 \mu \mathrm{s}$. The flight time of the molecules through the interaction region is inversely proportional to the molecular beam speed. The latter can be varied by using different carrier gases; i.e., the heavier the carrier gas the lower the speed. In the

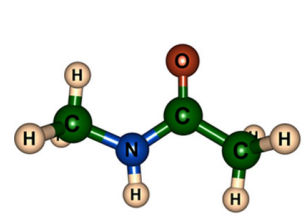

(a)

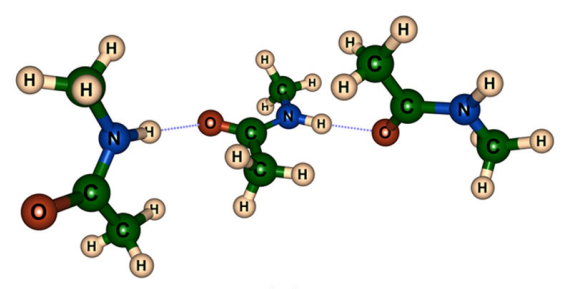

(c)

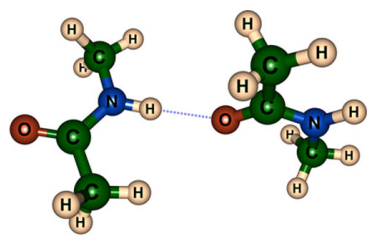

(b)
FIG. 2. The optimized structures of NMA oligomers: transmonomer (a), dimer (b), and trimer (c). The dashed lines depict hydrogen bonds $\mathrm{CO} \cdots \mathrm{HN}$. The structures were optimized with a B3LYP density functional [24] and a 6-31 $++G(d, p)$ basis set.

present study, we used Ar gas as the carrier for which the estimated time spent by the molecules in the interaction volume is $3 \mu \mathrm{s}$. The FELIX light was scanned in the wavelength range of $1000-1800 \mathrm{~cm}^{-1}$ and was used to ignite the MPD reactions in NMA. The NMA dissociation products were probed with VUV photons at $10.49 \mathrm{eV}$. The VUV light pulses were obtained as the third harmonic of a $355 \mathrm{~nm} \mathrm{Nd}^{3+}$ :YAG laser beam (Spectra Physics) in a gas cell containing a mixture of $\mathrm{Xe}$ and $\mathrm{Ar}$ gases at the ratio of $1: 10$. The ionized MPD products were detected with a time-of-flight reflectron-type mass spectrometer. To allow the longest possible interaction time, the VUV pulse entered the interaction volume just at the end of the mid-IR macropulse.

Figure 3 presents the mass spectrum obtained by irradiating the NMA sample with FELIX light at the wavelength of $1708 \mathrm{~cm}^{-1}$ as well as the mass spectrum measured when FELIX was off. As follows from the figure, the VUV photons alone produce predominantly NMA parent ions $(m / z=73)$. Note that the peak at $m / z=40$ results from the ionization of Ar seed gas. In contrast, the FELIX + VUV mass spectrum shows a variety of additional fragments, which are produced by mid-IR MPD and ionized afterwards with the VUV pulse (see, e.g., the peaks at $m / z=30,31,45$, and 58 in Fig. 3). Thus, backgroundfree IR spectra can be obtained in the sense that the fragments only appear upon resonant excitation with IR photons while they are absent without IR photons. This makes our measurements insensitive to fluctuations in the molecular source output, which is the essential advantage of this technique. In this way the mid-IR spectrum of NMA was measured, and is shown in Fig. 4(a), where the yield of the individual fragments as well as the total fragment yield is shown versus IR wavelength. The fragments' counts 


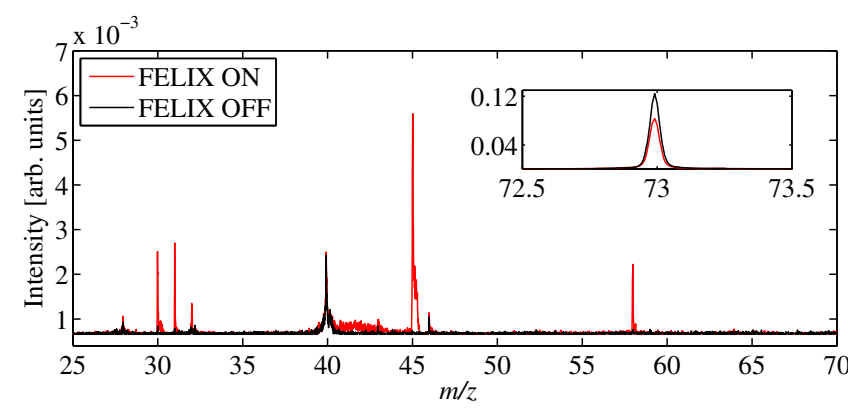

FIG. 3. The mass spectra of NMA obtained with VUV ionization $(10.49 \mathrm{eV})$. The red curve represents the spectrum when the NMA molecules prior to the VUV pulse were additionally irradiated with $1708 \mathrm{~cm}^{-1}$ FELIX light during several microseconds. The inset shows a part of the spectra corresponding to the parent molecular ion. The peak at $m / z=40$ corresponds to the $\mathrm{Ar}^{+}$ions created by two-photon VUV ionization.

were divided by the total ion yield $\left(I_{\text {parent }}+\Sigma I_{\text {fragments }}\right)$ and the laser power at the corresponding wavelengths.

In the FELIX + VUV mass spectrum a relatively strong peak appears at $m / z=45$ (see the red curve in Fig. 3), which cannot be produced by the direct breaking of one or several chemical bonds, but rather involves significant molecular rearrangement. In order to identify the origin of the $m / z=45$ fragment, we performed auxiliary measurements with the NMA- $d 7$ isotopologue in which all hydrogen atoms are substituted with deuterium atoms. In the FELIX + VUV mass spectrum of NMA- $d 7$ the $m / z=45$ fragment is shifted to $m / z=52$, which enables us to conclude that this fragment is associated with the formation of di-methylamine, $\mathrm{CH}_{3} \mathrm{NHCH}_{3}$, or ethylamine, $\mathrm{CH}_{3} \mathrm{CH}_{2} \mathrm{NH}_{2}$. It is also interesting to note that the corresponding fragments in the NMA- $d 7$ and NMA spectra have different intensities (see the Supplemental Material [25]). For example, the fragment associated with the peptide bond cleavage, $\mathrm{CH}_{3} \mathrm{CO}^{+}(\mathrm{m} / z=43)$, is almost not present in the NMA mass spectrum while $\mathrm{CD}_{3} \mathrm{CO}^{+}$ $(m / z=46)$ appears for NMA- $d 7$. Furthermore, the relative intensity of the fragment involving significant molecular geometry rearrangement $(\mathrm{m} / z=45$ for NMA and $m / z=$ 52 for NMA- $d 7$ ) reduces for the case of NMA- $d 7$. We believe that such behavior is attributed to the lower mobility (higher mass) of the deuterium atoms compared with hydrogen atoms; i.e., for the NMA- $d 7$ case the dissociation channels relying on molecular rearrangement are less probable, while the channels involving direct chemical bond ruptures become more significant.

Within this new technique, the mid-IR spectrum can also be recorded by monitoring the parent ion yield, but in contrast to the fragments, the absorption of mid-IR photons leads to a reduced yield (the inset in Fig. 3). As a result, the ion-dip spectrum can be obtained, which however is not background free. Interestingly, as follows from the inset, the parent ion signal reduces noticeably after resonant IR

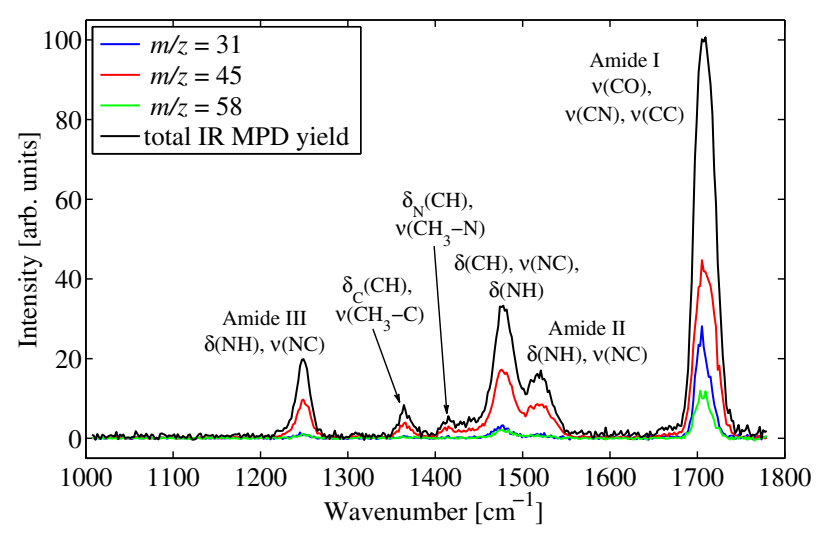

(a)

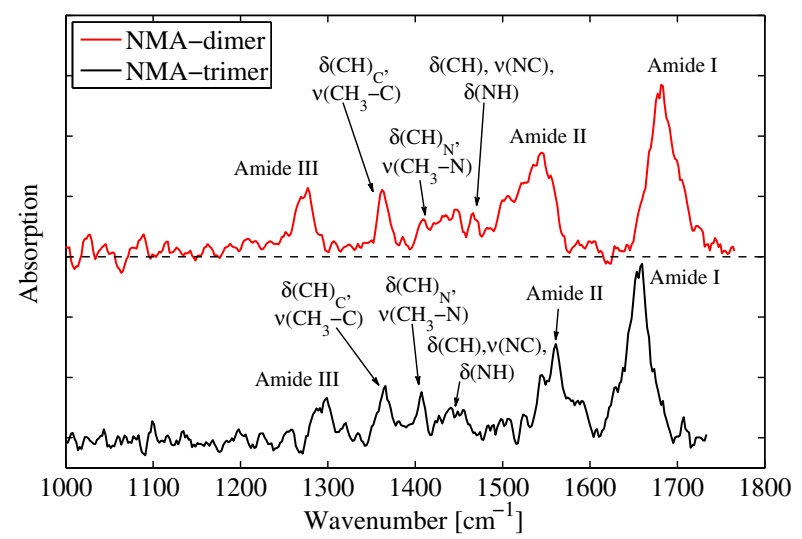

(b)

FIG. 4. (a) The IR spectra of NMA obtained by monitoring the yield of the most intense MPD fragments. All fragment yields were divided by the total ion yield $\left(I_{\text {parent }}+\Sigma I_{\text {fragments }}\right)$ and the laser power at the corresponding wavelengths. (b) The mid-IR spectra obtained by ion-dip measurements for the two NMA oligomers: dimers and trimers. The symbols $\delta$ and $\nu$ denote bending and stretching vibrations, respectively.

irradiation, implying that a significant fraction of the NMA molecules undergo MPD.

The measured IR spectrum of NMA [Fig. 4(a)] shows the vibrational transitions, which can be attributed to transNMA [Fig. 2(a)], the lowest energy structure. The strongest peaks correspond to amide I-III vibrations and the deformations of methyl groups, in agreement with the theoretical interpretation available in the literature [26,27]. The amide I-III bands were observed at 1707, 1519, and $1248 \mathrm{~cm}^{-1}$, respectively. The strongest methyl deformation bands were observed at 1478, 1415, and $1364 \mathrm{~cm}^{-1}$ [see Fig. 4(a) for the assignment]. The width of the IR peaks is about $1.1 \%-1.5 \%$ FWHM, slightly larger than the $1 \%$ bandwidth of the FELIX radiation. We did not observe the vibrational features associated with cis-NMA, which can be explained by the fact that the energy of this structure lies significantly higher than that of trans-NMA, leading to a negligible population of cis-NMA in the cooled molecular beam.

Apart from the advantage of background-free IR measurements, the suggested experimental scheme also enables 
the simultaneous measurement of IR spectra for several species present in the target beam, for example, oligomers of the parent molecules. Figure 4(b) presents the spectra of the NMA dimers and trimers measured simultaneously with the IR MPD spectrum of the NMA monomer. The spectra were obtained by monitoring the parent dimer and trimer ion yield versus the IR wavelength and, thus, represent the ion-dip spectra. The dips, in this case, are associated with the breaking of weak hydrogen bonds (the dashed lines in Fig. 2), through which the NMA molecules are coupled together to form NMA dimers and trimers.

The measured experimental gas-phase spectra of the NMA oligomers, not previously accessible by other experimental techniques in the full $1000-1800 \mathrm{~cm}^{-1}$ range [28], reveal some common spectral shifts in the positions of the amide I-III bands. The shifts are associated with the formation of the $\mathrm{CO} \cdots \mathrm{HN}$ hydrogen bonds that stabilize the structures of the NMA oligomers. Amide I corresponds to the $\mathrm{CO}$ stretch vibration with some $\mathrm{CN}$ and $\mathrm{CC}$ stretch, and is located at 1708,1681 , and $1656 \mathrm{~cm}^{-1}$ for the NMA monomers, dimers, and trimers, respectively. The redshift observed for the oligomers with respect to the monomer is attributed to the more delocalized nature of the $\mathrm{CO}$ stretch oscillators due to the $\mathrm{CO}$ bond length elongation upon hydrogen bonding $[29,30]$. It is worth noting that the shoulder of the amide I band at $1702 \mathrm{~cm}^{-1}$ in the spectrum of the NMA dimers is due to the non-hydrogen-bonded CO stretch vibration [see Fig. 2(b)]. Its frequency is only slightly redshifted by $6 \mathrm{~cm}^{-1}$ with respect to the monomer frequency due to the $\mathrm{NH}$ group being hydrogen bonded [29]. In contrast to the amide I band, the positions of the amide II band for the NMA oligomers are blueshifted with respect to the monomer. The frequency blueshift is due to the combination of the two effects of hydrogen bonding: the geometry change due to the elongation of $\mathrm{NH}$ and $\mathrm{C}-\mathrm{N}$ bonds, and the electron density redistribution on the carbonyl oxygen and amide hydrogen atoms [29,30]. The same factors lead to the blueshift in the frequencies of the amide III bands, which we observed at 1277 and $1298 \mathrm{~cm}^{-1}$ for the NMA dimers and trimers, respectively.

In this Letter we demonstrate a new action spectroscopy method for measuring background-free IR spectra for neutral isolated molecules of arbitrary structure. This approach significantly extends the scope of the molecules that can be studied with IR spectroscopy in the gas phase. In particular, it is suitable for molecules lacking an UV chromophore. This new method is demonstrated here for the mid-IR range, and is readily applicable to measure spectra in the far-IR and terahertz range under the condition that the corresponding far-IR and terahertz vibrational modes are characterized by fast IVR rates. The method we suggest is not only limited to small molecules but can be applied to all biomolecules that can be delivered to the gas phase with the help of the laser desorption technique [6,31]. In the field of gas-phase IR spectroscopy of biomolecules
$[4,32,33]$, the interactions responsible for local structural motifs as well as those induced by neighboring molecules are elucidated. Such studies are particularly important for gaining insight into the relations between the properties of biomolecules and their structures. The method demonstrated is highly suitable for this research field, since it enables the study of molecular associates, such as microhydrated molecules [34], and one can selectively study the intrinsic properties of biomolecules associated with intramolecular interactions as well as those associated with interactions and perturbations induced by the ambient environment (intermolecular interactions). We believe that the technique presented in this work will significantly advance the field of molecular structure characterization.

This work was supported by the Swedish Research Council (VR), the Knut and Alice Wallenberg Foundation (KAW), Sweden, and the European Community's Seventh Framework Programme (FP7/ 2007-2013) under Grant Agreement No. 312284. P. S. acknowledges financial support from the StockholmUppsala Center for Free Electron Laser Research.

*a.rijs@science.ru.nl

†vitali.zhaunerchyk@physics.gu.se

[1] C. Soto, Unfolding the role of protein misfolding in neurodegenerative diseases, Nat. Rev. Neurosci. 4, 49 (2003).

[2] Y. S. Eisele, C. Monteiro, C. Fearns, S. E. Encalada, R. L. Wiseman, E. T. Powers, and J. W. Kelly, Targeting protein aggregation for the treatment of degenerative diseases, Nat. Rev. Drug Discovery 14, 759 (2015).

[3] R. Weinkauf, J.-P. Schermann, M. de Vries, and K. Kleinermanns, Molecular physics of building blocks of life under isolated or defined conditions, Eur. Phys. J. D 20, 309 (2002).

[4] See Gas-Phase IR Spectroscopy and Structure of Biological Molecules, Topics in Current Chemistry Vol. 364, edited by A. M. Rijs and J. Oomens (Springer International Publishing, Switzerland, 2015), pp. 1-406, and references therein.

[5] R. J. Falconer and A. G. Markelz, Terahertz spectroscopic analysis of peptides and proteins, J. Infrared, Millimeter, Terahertz Waves 33, 973 (2012).

[6] M. S. de Vries and P. Hobza, Gas-Phase spectroscopy of biomolecular building blocks, Annu. Rev. Phys. Chem. 58, 585 (2007).

[7] D. F. Plusquellic, K. Siegrist, E. J. Heilweil, and O. Esenturk, Applications of Terahertz spectroscopy in biosystems, Chem. Phys. Chem. 8, 2412 (2007).

[8] L. Quaroni and T. Zlateva, Infrared spectromicroscopy of biochemistry in functional single cells, Analyst 136, 3219 (2011).

[9] V. Yatsyna, D. J. Bakker, R. Feifel, A. M. Rijs, and V. Zhaunerchyk, Aminophenol isomers unraveled by conformer-specific far-IR action spectroscopy, Phys. Chem. Chem. Phys. 18, 6275 (2016). 
[10] S. Jaeqx, J. Oomens, A. Cimas, M.-P. Gaigeot, and A. M. Rijs, Gas-Phase peptide structures unraveled by far-IR spectroscopy: Combining IR-UV ion-dip experiments with Born-Oppenheimer molecular dynamics simulations, Angew. Chem., Int. Ed. 53, 3663 (2014).

[11] D. J. Bakker, A. Peters, V. Yatsyna, V. Zhaunerchyk, and A. M. Rijs, Far-infrared signatures of hydrogen bonding in phenol derivatives, J. Phys. Chem. Lett. 7, 1238 (2016).

[12] F. Kollipost, J. Andersen, D. W. Mahler, J. Heimdal, M. Heger, M. A. Suhm, and R. Wugt Larsen, The effect of hydrogen bonding on torsional dynamics: A combined far-infrared jet and matrix isolation study of methanol dimer, J. Phys. Chem. Lett. 141, 174314 (2014).

[13] A. van der Meer, FELs, nice toys or efficient tools?, Nucl. Instrum. Methods Phys. Res., Sect. A 528, 8 (2004); http:// www.ru.nl/felix/.

[14] F. Glotin, J. Ortega, R. Prazeres, and C. Rippon, Activities of the CLIO infrared facility, Nucl. Instrum. Methods Phys. Res., Sect. B 144, 8 (1998).

[15] J. Campbell, G. Hancock, J. Halpern, and K. Welge, Dissociation of $\mathrm{NH}_{3}$ to electronic ground-state fragments by high-intensity $\mathrm{CO}_{2}$ laser radiation, Opt. Commun. 17, 38 (1976).

[16] D. S. Bomse, R. L. Woodin, and J. L. Beauchamp, Molecular activation with low-intensity $\mathrm{CW}$ infrared laser radiation. Multiphoton dissociation of ions derived from diethyl ether, J. Am. Chem. Soc. 101, 5503 (1979).

[17] S. Mukamel and J. Jortner, Multiphoton molecular dissociation in intense laser fields, J. Chem. Phys. 65, 5204 (1976).

[18] J. Oomens, B. G. Sartakov, G. Meijer, and G. von Helden, Gas-phase infrared multiple photon dissociation spectroscopy of mass-selected molecular ions, Int. J. Mass Spectrom. 254, 1 (2006).

[19] J. R. Eyler, Infrared multiple photon dissociation spectroscopy of ions in Penning traps, Mass Spectrom. Rev. 28, 448 (2009).

[20] J. Oomens, N. Polfer, D. T. Moore, L. van der Meer, A. G. Marshall, J. R. Eyler, G. Meijer, and G. von Helden, Chargestate resolved mid-infrared spectroscopy of a gas-phase protein, Phys. Chem. Chem. Phys. 7, 1345 (2005).

[21] Y. Fung, T. Besson, J. Lemaire, P. Maitre, and R. Zubarev, Room-temperature infrared spectroscopy combined with mass spectrometry distinguishes gas-phase protein isomers, Angew. Chem., Int. Ed. 48, 8340 (2009).

[22] J. T. O'Brien, J. S. Prell, G. Berden, J. Oomens, and E.R. Williams, Effects of anions on the zwitterion stability of Glu, His and Arg investigated by IRMPD spectroscopy and theory, Int. J. Mass Spectrom. 297, 116 (2010).

[23] A. M. Rijs and J. Oomens, IR Spectroscopic Techniques to Study Isolated Biomolecules, Gas-Phase IR Spectroscopy and Structure of Biological Molecules, edited by A. M. Rijs and J. Oomens (Springer International Publishing, Switzerland, 2015), pp. 1-42.

[24] A. D. Becke, Density-functional thermochemistry. III. The role of exact exchange, J. Chem. Phys. 98, 5648 (1993).

[25] See Supplemental Material at http://link.aps.org/ supplemental/10.1103/PhysRevLett.117.118101 for the mass spectra produced by IR multiphoton dissociation of NMA- $d 7$, and the mass spectra of the NMA oligomers.

[26] A. L. Kaledin and J. M. Bowman, Full dimensional quantum calculations of vibrational energies of N-methyl acetamide, J. Phys. Chem. A 111, 5593 (2007).

[27] M. P. Gaigeot, R. Vuilleumier, M. Sprik, and D. Borgis, Infrared spectroscopy of $\mathrm{N}$-methylacetamide revisited by ab initio molecular dynamics simulations, J. Chem. Theory Comput. 1, 772 (2005).

[28] M. Albrecht, C. A. Rice, and M. A. Suhm, Elementary peptide motifs in the gas Phase: FTIR aggregation Study of formamide, acetamide, N-methylformamide, and N-methylacetamide, J. Phys. Chem. A 112, 7530 (2008).

[29] N. S. Myshakina, Z. Ahmed, and S. A. Asher, Dependence of amide vibrations on hydrogen bonding, J. Phys. Chem. B 112, 11873 (2008).

[30] A. M. Rijs, I. Compagnon, J. Oomens, J. S. Hannam, D. A. Leigh, and W. J. Buma, Stiff, and sticky in the right places: Binding interactions in isolated mechanically interlocked molecules probed by mid-infrared spectroscopy, J. Am. Chem. Soc. 131, 2428 (2009).

[31] D. S. Peterson, Matrix-free methods for laser desorption/ ionization mass spectrometry, Mass Spectrom. Rev. 26, 19 (2007).

[32] J.-P. Schermann, Spectroscopy and Modeling of Biomolecular Building Blocks (Elsevier, Amsterdam, 2008).

[33] N. S. Nagornova, T. R. Rizzo, and O. V. Boyarkin, Interplay of intra- and intermolecular $\mathrm{H}$-Bonding in a progressively solvated macrocyclic peptide, Science 336, 320 (2012).

[34] M. Cirtog, A. M. Rijs, Y. Loquais, V. Brenner, B. Tardivel, E. Gloaguen, and M. Mons, Far/Mid-infrared signatures of solvent-solute interactions in a microhydrated model peptide chain, J. Phys. Chem. Lett. 3, 3307 (2012). 